\title{
ANÁLISE DE REGIMES DE ESCOAMENTO, SATURAÇÃO DE GÁS E CAPACITÂNCIA EM COLUNA BOLHA
}

\author{
Luciana Bennemann Potrich ${ }^{1}$
}

Resumo: Este trabalho foi desenvolvido experimentalmente e tem como objetivo identificar os regimes de escoamento, a saturação de gás e o comportamento da capacitância do sistema. Foi possível identificar na coluna a presença dos regimes: homogêneo, transição e heterogêneo. Quanto à saturação de gás, observou-se que este parâmetro aumenta com o aumento da vazão de ar. Em relação às medidas de capacitância do sistema, verificaram-se padrões distintos de comportamento da capacitância em função do tempo para os diferentes regimes de escoamento obtidos, esse fato abre a possibilidade real de empregos de medida de capacitância em função do tempo para identificação dos regimes de escoamento.

Palavras chave: Dinâmica dos fluidos, capacitores, escoamento bifásico, gas holdup.

Abstract: This work was performed experimentally and aims to determine the flow regimes, gas holdup and the system capacitance behavior. The results indicated the presence of homogeneous regime, transition regime and heterogeneous regime. It was observed the increasing of gas holdup with increasing gas flow. For measures capacitance of the system, there were different patterns of capacitance versus time for different flow regimes obtained, this fact opens up the possibility of using actual measurements of capacitance versus time for identification of the flow regimes.

Keywords: Fluid dynamics, capacitor, biphasic flow, gas holdup.

\footnotetext{
${ }^{1}$ Universidade Estadual do Oeste do Paraná - UNIOESTE. Rua da Faculdade, 645. Jardim La Salle. CEP 85903-000. E-mail: luciana.potrich@ yahoo.com.br
} 


\section{INTRODUÇÃO}

Escoamentos bifásicos, normalmente encontrados em coluna bolha, ocorrem em diversos processos industriais como, por exemplo, em plantas nucleares, de exploração de petróleo e química (Cunha Filho, 2010). Eles são usados principalmente em processos químicos envolvendo reações tais como oxidação, cloração, alquilação, polimerização e hidrogenação na fabricação de combustíveis sintéticos por conversão de gás e em processos bioquímicos como fermentação e tratamento biológico de águas residuais (Kantarci et al, 2004).

Uma atenção especial vem sendo dada ao estudo do escoamento bifásico, quer seja pela crescente importância que vem sendo dada a esse assunto nos diversos ramos da engenharia, quer seja pela dificuldade de compreendê-lo. (Lira, 1980).

Embora essa operação seja vantajosa, devido à fácil operação, a fluidodinâmica da coluna bolha é complexa e depende das propriedades físicas do sistema (formato do distribuidor de gás, diâmetro da coluna, etc.).

O conhecimento dos fenômenos envolvidos nesses escoamentos é de grande importância para o desenvolvimento de equipamentos mais eficientes, seguros e econômicos. Dentre estes fenômenos pode-se citar saturação de gás e regimes de escoamento (Cunha Filho, 2010).

Reatores de coluna de bolha apresentam três tipos de regime de escoamento, chamados de homogêneo (bolha), transição e heterogêneo (turbulento). Basicamente, para cada sistema gás-líquido, a formação e a estabilidade destes regimes dependem das velocidades superficiais do gás e do líquido, do distribuidor de gás e da geometria do reator de borbulhamento (Silva, 2008).

O regime homogêneo é caracterizado por baixas velocidades superficiais de gás, onde o tamanho de bolhas é radialmente uniforme, sendo que as bolhas apresentam diâmetros pequenos e formas esféricas, além de possuírem pouca interação entre si.

Com o aumento da vazão de gás, o escoamento se torna instável e o regime homogêneo não consegue manter-se, passando por um estado de transição.

A transição ocorre em uma pequena faixa de velocidades superficiais do gás, onde bolhas maiores, formadas por coalescência, ascendem com uma velocidade maior que a das bolhas menores. Neste regime, um padrão de circulação do líquido começa a se desenvolver.

No ponto onde o escoamento tornase caótico é possível visualizar o regime heterogêneo, que é caracterizado por bolhas com diferentes formas e tamanhos e uma intensa circulação de líquido no interior da coluna (Ajbar et al, 2008).

Este estudo visa comprovar a existência dos regimes de escoamento apresentados na literatura para a coluna bolha construída para este trabalho e de maneira inovadora correlacioná-los por meio de medidas de capacitância do sistema, buscando complementar os estudos da fluidodinâmica de reatores coluna bolha.

Outro parâmetro que deve ser monitorado e controlado em coluna bolha é a fração das fases, isto é, as frações do volume ou da seção transversal ocupada por uma dada fase no escoamento bifásico. A saturação de gás (gas holdup) nada mais é que a fração volumétrica do gás contido na mistura binária da coluna. Conhecê-lo é de suma importância no projeto de colunas de bolhas por estar diretamente ligado ao fenômeno de transporte de massa entre as fases (Dionísio, 2008). Esse parâmetro depende da velocidade superficial do gás e essa dependência normalmente é na forma da equação (1).

$$
\varepsilon_{\mathrm{G}} \alpha U_{G}^{n}
$$

onde $\varepsilon_{\mathrm{G}}$ é a saturação de gás, $\mathrm{U}_{\mathrm{G}}$ é a velocidade superficial do gás e o fator $n$ depende do regime do fluxo. 
Segundo Shawaqfeh (2002), os valores de $n$ para regime homogêneo variam de 0,7 a 1,2 , enquanto para os regimes heterogêneos ou transição variaram de 0,4 à 0,7 .

Diferentes métodos de obtenção da saturação de gás encontram-se na literatura, sendo o mais utilizado, devido a sua simplicidade de execução, o método de drenagem, que consistem no fechamento simultâneo das válvulas de entrada e saída da coluna, com a posterior anotação das alturas de gás e líquido após separação completa das fases.

Ao longo dos anos uma variada gama de técnicas vem sendo desenvolvidas para medir parâmetros bifásicos, sendo o objetivo deste trabalho estudar os regimes de escoamento e tentalos relacionar a medidas de capacitância, e além disso, determinar a saturação de gás em coluna bolha.

\section{MATERIAIS E MÉTODOS}

\subsection{Materiais}

O módulo experimental, como mostrado na Figura 1, é composto por um leito de bolhas com altura de $100 \mathrm{~cm}$ e diâmetro de $5 \mathrm{~cm}$, construído em acrílico para possibilitar a visualização dos regimes de escoamento. Como fluidos foram utilizados o ar e a água.

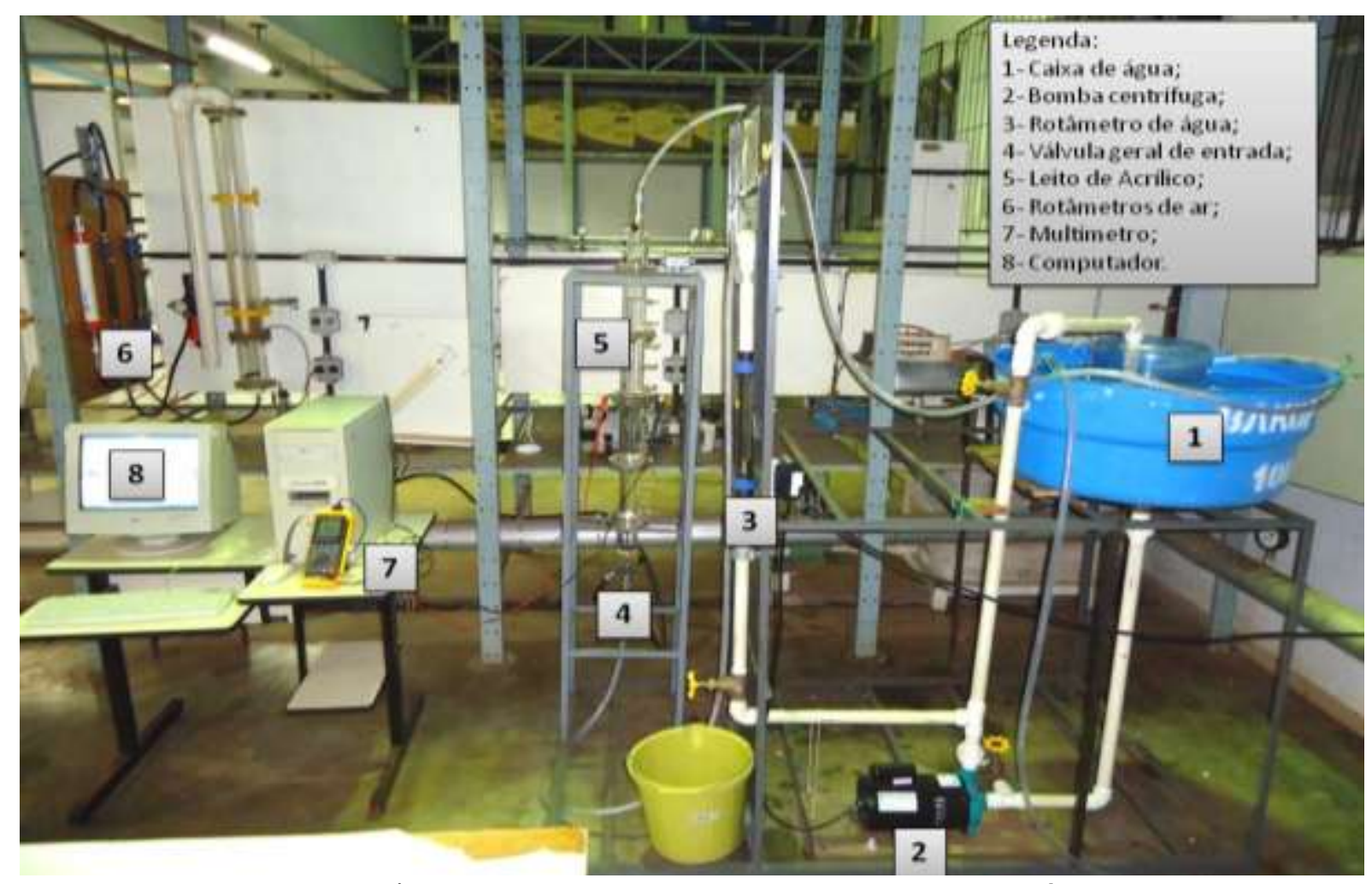

Figura 1- Módulo experimental para o estudo fluidodinâmico.

A alimentação de ar foi feita através de uma linha contendo um compressor, uma válvula reguladora de pressão e um rotâmetro para controle da vazão de ar. A linha de água sai de uma caixa d'água, passando por uma bomba centrífuga de $1 \mathrm{HP}$ e por um rotâmetro que mede a vazão de água. Por meio de uma fonte pontual, os fluidos são misturados previamente e inseridos no leito.
Os capacitores, como mostrado na Figura 2, são constituídos por placas de alumínio, inseridos por meio de flanges no leito, a fim de, ficarem na posição central do leito, para melhor coleta dos dados. Para este estudo foram desprezados as influencias da forma e posição dos capacitores dentro do leito. 
Estes capacitores encontram-se ligados a um multímetro, que transfere as medidas ao computador.

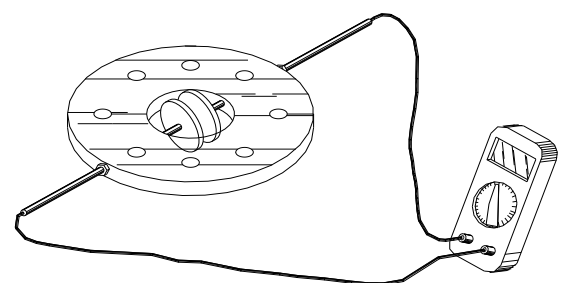

Figura 2 - Esquema do capacitor.

\subsection{Métodos}

Identificação dos regimes de escoamento: Os regimes foram identificados visualmente de acordo com a metodologia de Moreira e Freire (2003). Este procedimento foi realizado por meio de diversas combinações de vazões águaar, esperando-se 1 min para a completa estabilização do escoamento.

Medidas de capacitância: Inicialmente, verificou-se a influência da temperatura e da vazão nas medidas de capacitância. A partir deste estudo prévio, fez-se o registro da capacitância em função do tempo. Monitorou-se durante 2 minutos para cada medida, fixando-se uma vazão de água e variando-se a vazão de ar, a fim de obter as medidas para o sistema bifásico.

Medidas de saturação de gás: Utilizou-se o método de drenagem para determinação do parâmetro saturação. Este procedimento foi realizado através do fechamento simultâneo das válvulas de entrada e de saída da coluna, medindo-se com uma régua a altura de água dentro da coluna após seu repouso, em relação altura total do sistema, sendo seu valor obtido usando a equação 2:

$$
\varepsilon_{G}=1-\frac{\text { altura }_{\text {água }}}{\text { altura }_{\text {Total }}}
$$

\section{RESULTADOS}

\subsection{Identificação dos regimes de escoamento}

A Figura 3 apresenta o "mapa" de regimes de escoamento obtidos experimentalmente em função das vazões de ar e de água utilizadas neste trabalho. A partir do qual, é possível identificar as fronteiras de cada regime, ou seja, o par ordenado de vazões encontrado para cada tipo de escoamento.

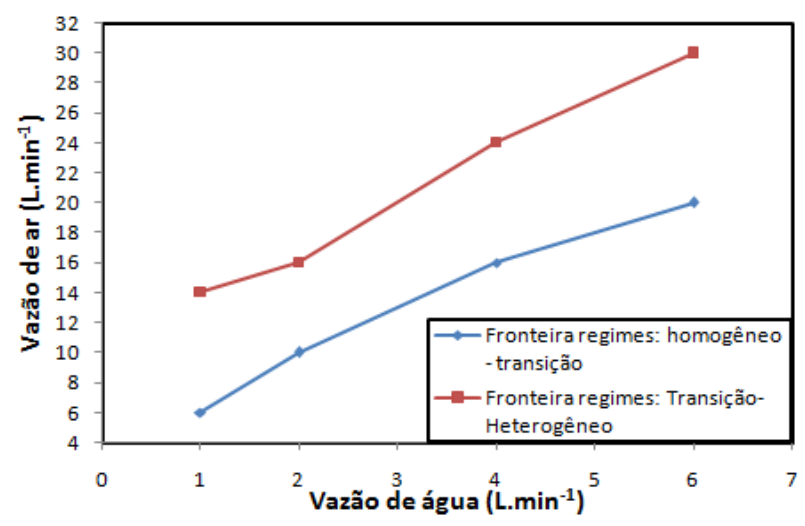

Figura 3 - Mapa de escoamentos

Cabe salientar que este mapa de escoamentos só é válido para colunas com as mesmas dimensões da utilizada neste experimento.

A literatura cita diferentes métodos para o reconhecimento dos padrões de escoamentos bifásicos. Sendo, a técnica mais comum e simples, a observação visual diretamente do escoamento através de um tubo transparente ou visor. Neste experimento, verificou-se os três regimes de escoamento identificados por Silva (2008) em coluna de bolha: homogêneo, transição e heterogêneo (Figura 4).

A Figura 4 apresenta um comparativo entre os três regimes obtidos, e observamos que à baixas vazões de ar foi obtido o regime homogêneo, para altas vazões de ar o escoamento tornou-se completamente desordenado e com bolhas de ar em tamanho maior, caracterizando o regime heterogêneo. Entre esses regimes, ocorreu o regime de transição, o qual é de difícil visualização, pois possui características dos dois regimes citados anteriormente. À medida que a vazão de água aumenta a fronteira do regime 
homogêneo - transição se desloca ascendentemente, possibilitando trabalhar em regime homogêneo com altas vazões de ar.

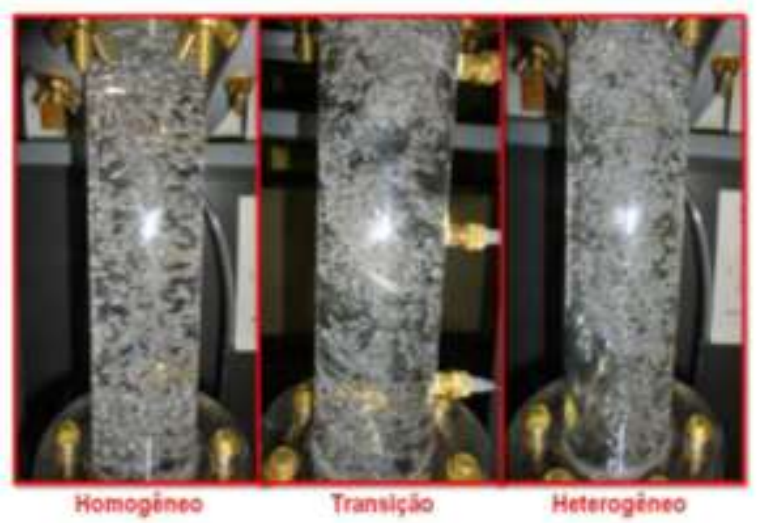

Figura 4 - Regimes de escoamento encontrados.

\subsection{Medidas de capacitância do sistema}

O método capacitivo utilizado neste experimento possui seu funcionamento baseado na diferença entre as constantes dielétricas das fases.

A primeira etapa realizada foi o reconhecimento das possíveis influências que o sistema poderia vir a sofrer. Quanto à variação da vazão de água e da temperatura do sistema. Através de testes estatísticos foi possível quantificar cada influência. Foram utilizados o teste $t$, que consiste em um teste de hipótese onde rejeita-se ou não uma hipótese nula, e pvalor, que é a probabilidade de obter o efeito estudado quando a hipótese nula é verdadeira. (MOORE, 2000),

Para realização destes testes estatísticos foi utilizado um nível de significância de $\alpha=5 \%$. As hipóteses nula e alternativa foram estipuladas por tratarem de igualdade das médias e diferença entre médias, respectivamente.

Para o teste de influência de temperatura retirou-se dados de capacitância em duas temperaturas, $27^{\circ} \mathrm{C}$ e $31^{\circ} \mathrm{C}$.

A Tabela 1 apresenta os valores de $\mathrm{t}$ e p-valor para cada vazão analisada.
Tabela 1: Testes estatísticos realizados para verificação da influência da temperatura

\begin{tabular}{|c|c|c|c|c|}
\hline \multirow{2}{*}{$\begin{array}{c}\text { Vazão } \\
(\mathrm{L} . \\
\left.\text { min }^{-1}\right)\end{array}$} & \multicolumn{2}{|c|}{$\begin{array}{l}\text { Capacitância } \\
\text { Média }(\mu \mathrm{F})\end{array}$} & \multirow{2}{*}{$\begin{array}{c}\text { Teste } \\
\mathbf{t}\end{array}$} & \multirow[t]{2}{*}{ p-valor } \\
\hline & $27^{\circ} \mathrm{C}$ & $31^{\circ} \mathrm{C}$ & & \\
\hline 1 & 25,24 & 28,84 & 9,65 & $7,68 \mathrm{E}-17$ \\
\hline 2 & 25,69 & 29,12 & 8,88 & $1,53 \mathrm{E}-14$ \\
\hline 4 & 26,09 & 29,35 & 13,40 & $5,49 \mathrm{E}-29$ \\
\hline 6 & 26,25 & 29,42 & 13,62 & $9,72 \mathrm{E}-30$ \\
\hline
\end{tabular}

O valor tabelado para $\mathrm{t}$, de acordo com os graus de liberdade, é de 1,96 (Moore, 2000). Verifica-se então que a temperatura tem grande influência sobre as medidas de capacitância, já que o valor de $\mathrm{t}$ calculado é maior que o tabelado. $\mathrm{O}$ p-valor também mostrou-se significativo contra a hipótese nula, pois quanto menor for este valor comparado a $\alpha$, mais significante é a influência da variável, ou seja, pode-se afirmar que a hipótese nula (médias iguais) é rejeitada com nível de confiança de $95 \%$.

Para a influência da vazão os mesmos testes estatísticos foram realizados, porém não foi encontrada influência significativa.

Realizados os testes preliminares, o experimento passou a ser realizado em uma temperatura fixa de $27^{\circ} \mathrm{C}$, para que o sistema não sofresse qualquer influência.

Paralelamente à identificação visual dos regimes, foi realizada as medidas de capacitância em duplicata. A Figura 5 apresenta um comparativo entre as capacitâncias dos três regimes encontrados, para a vazão fixada de água de $1 \mathrm{~L} / \mathrm{min}$.

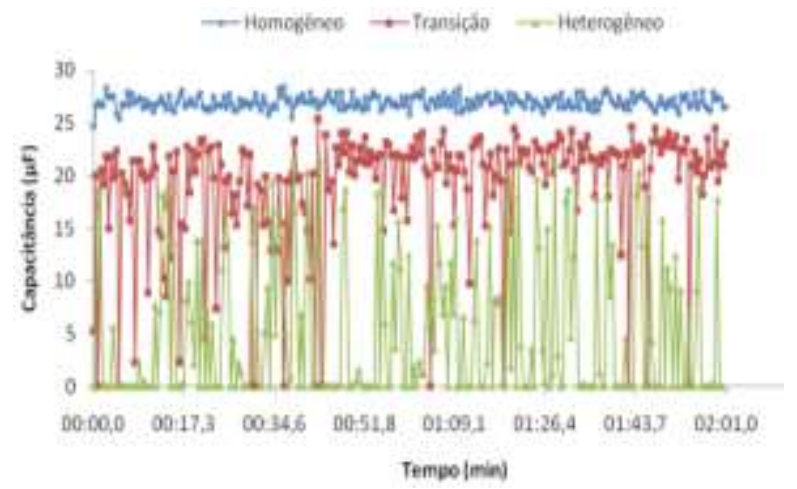

Figura 5 - Capacitância versus tempo explicitando o comportamento dos regimes de escoamento. 
É possível visualizar que o regime homogêneo traz pouca variação em suas medidas. Esta variação aumenta gradativamente com a mudança de regime, chegando ao regime heterogêneo com um desvio muito grande entre as medidas.

Complementando a Figura 5, a Tabela 2 apresenta as médias e o desvio padrão das medidas referentes às fronteiras de cada regime.

Tabela 2: Média e desvio padrão de cada regime de escoamento encontrado no experimento - vazão de água fixada em 1 L.min ${ }^{-1}$.

\begin{tabular}{cccc}
\hline $\begin{array}{c}\text { Vazão ar } \\
(\text { L.min }\end{array}$,, & Regime & $\begin{array}{c}\text { Média } \\
(\boldsymbol{\mu F})\end{array}$ & $\begin{array}{c}\text { Desvio } \\
\text { Padrão }\end{array}$ \\
\hline 2 & Homogêneo & 31,80 & 0,20 \\
6 & Homogêneo & 26,53 & 0,53 \\
8 & Transição & 24,48 & 1,46 \\
12 & Transição & 18,99 & 5,96 \\
14 & Heterogêneo & 14,61 & 9,52 \\
20 & Heterogêneo & 7,09 & 9,02 \\
\hline
\end{tabular}

Observando-se a Tabela 2, torna-se mais evidente à existência de um padrão de comportamento na capacitância para cada regime de escoamento. A capacitância foi testada em maiores vazões de água, e em diferentes pontos da coluna, e verificou-se o mesmo padrão, com pouco desvio entre medidas para o regime homogêneo, aumentando conforme o aumento da vazão de ar.

Essa diferença entre os padrões no comportamento da capacitância em cada regimes de escoamento pode ser explicada pelas constantes dielétricas da água e do ar. A constante dielétrica $\mathrm{K}$ do ar encontra-se em torno de 1,0001, já a da água é de 78,0 (Mussoi e Villaça, 2000).

Essa grande diferença entre os valores das constantes faz com que as leituras de capacitância variem de acordo com o fluxo de água e ar na coluna.

Quanto maior for a vazão de ar na coluna, menor será a capacitância média do sistema. Também, quanto mais desordenado for o fluxo, maior será o desvio entre as medidas de capacitância.
Este comportamento pode ser comprovado por meio do experimento realizado, onde o regime homogêneo apresentou poucos picos de leitura e um valor alto de capacitância. Com a mudança de escoamento, houve um aumento do número de picos das leituras e uma diminuição da capacitância média do sistema.

Os resultados indicam a possibilidade do emprego das medidas de capacitância para identificar os regimes, tornando possível a implantação de um sistema de controle de escoamento em reatores de coluna bolha. Trabalhar em um regime fixo é de extrema importância para que as variáveis controladas se mantenham o mais próxima possível das condições ótimas do processo, e isso só é possível com a implantação de um sistema de controle de processos.

\subsection{Saturação de gás}

Muitos estudos da literatura vêm relacionando velocidade superficial de gás com a saturação do gás. Schumpe e Deckwer (1982) reportaram uma equação similar à equação (1), empregando uma solução de carboximetilcelulose, como mostrado na equação (3).

$$
\varepsilon_{G}=a U_{G}^{b}
$$

Akita e Yoshida (1973) relataram uma forma funcional empírica para a saturação de gás, justificando ser válida para diferentes regimes de fluxo (equação 4).

$$
\varepsilon_{G}=\frac{U_{G}}{a+b U_{G}}
$$

A Tabela 3 mostra as constantes das equações (3) e (4) utilizadas pelos autores.

Tabela 3: Valores das constantes das equações (3) e (4)

\begin{tabular}{ccc}
\hline Grupo & \multicolumn{2}{c}{ Constantes } \\
& $\mathbf{a}$ & $\mathbf{b}$ \\
\hline Eq. (3) & 0,04 & 0,63 \\
Eq. (4) & 0,29 & 1,80 \\
\hline
\end{tabular}


Os valores de saturação de ar, obtidos a partir da Equação 2, estão apresentados na Figura 6 como uma função da velocidade superficial do ar, testadas em diferentes vazões de água. A figura ainda apresenta os valores calculados com as correlações utilizadas.

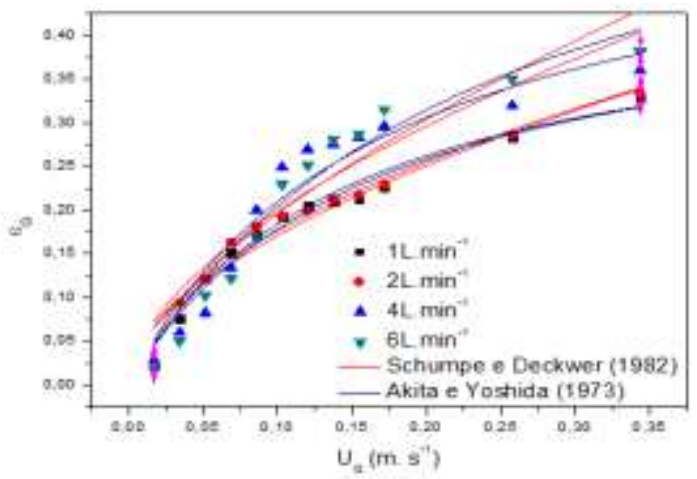

Figura 6 - Variação da saturação de gás $\left(\varepsilon_{G}\right)$ com a velocidade superficial do $\operatorname{ar}\left(\mathbf{U}_{\mathbf{G}}\right)$, testado a diferentes vazões de água.

Observando-se a Figura 6 é possível verificar que a influência da vazão de água não é tão significativa na mudança de 1 para $2 \operatorname{Lmin}^{-1}$ e de 4 para $6 \mathrm{Lmin}^{-1}$, ou seja, para pequenas mudanças nessa vazão não ocorrem modificações expressivas na saturação de ar. Resultado condizente com o obtido por Shawaqfeh (2002).

A Tabela 4 apresenta os ajustes dos dados de saturação de gás para as diferentes correlações testadas (equações 3 e 4).

Tabela 4: Ajustes dos dados de saturação de gás para diferentes correlações

\begin{tabular}{ccccc}
\hline Correlação & $\begin{array}{c}\text { Vazão de } \\
\mathbf{H}_{2} \mathbf{O}\end{array}$ & \multicolumn{3}{c}{ Constantes } \\
& (L.min $^{-1}$ ) & $\mathbf{a}$ & $\mathbf{b}$ & $\mathbf{r}^{\mathbf{2}}$ \\
\hline \multirow{3}{*}{ Eq. (3) } & 1 & 0,61 & 0,55 & 0,95 \\
& 2 & 0,60 & 0,53 & 0,95 \\
& 4 & 0,74 & 0,57 & 0,85 \\
& 6 & 0,85 & 0,64 & 0,90 \\
\hline \multirow{5}{*}{ Eq. (4) } & 1 & 0,34 & 2,14 & 0,97 \\
& 2 & 0,32 & 2,19 & 0,97 \\
& 4 & 0,30 & 1,76 & 0,92 \\
& 6 & 0,34 & 1,46 & 0,95 \\
\hline
\end{tabular}

Observa-se na Tabela 4 que o ajuste de Schumpe e Deckwer (eq.3) obteve os menores valores de $r^{2}$. Isto se deve ao fato da fase fluida deste experimento ser diferente da obtida pelo autor em seus estudos, conforme relatado anteriormente. Já o ajuste de Akita e Yoshida (eq.4) obteve valores maiores de $\mathrm{r}^{2}$, e os valores das constantes $\mathrm{a} e \mathrm{~b}$ aproximaram-se das relatadas pelo autor, mostrando que os dados do experimento se ajustam melhor a esta equação, principalmente quando se trabalha com baixas vazões de água.

\section{CONCLUSÃO}

Por meio das determinações dos regimes de escoamento, saturação de gás em função das vazões de gás e líquido e o comportamento da capacitância do sistema, foram possíveis obter três conclusões significativas.

Identificou-se a presença de três regimes distintos de escoamento para as vazões de ar e de água utilizadas neste estudo: regime homogêneo, transição e heterogêneo e determinou-se a faixa de vazões de ar e água de cada regime.

Quanto à saturação de gás, verificou-se que a mesma aumenta com o aumento da vazão de ar. Já com a vazão de água dois comportamentos foram encontrados. Até uma vazão de ar de 0,07 L. $\min ^{-1}$, um aumento da vazão de água resultou em uma diminuição da saturação de gás. Acima deste valor inverteu-se este comportamento, um aumento na vazão de água fez com que também aumentasse a saturação de gás.

Analisando as medidas de capacitância do sistema, verificaram-se padrões distintos de comportamento da capacitância em função do tempo para os diferentes regimes de escoamento estudados. A mudança no padrão está diretamente relacionada ao desvio-padrão da medida. Esse fato abre a possibilidade real de emprego das medidas de capacitância em função do tempo para a identificação dos regimes de escoamento.

Manter o regime fixo é importante para que se mantenham as condições 
ótimas do processo, e isso só é possível com a implantação de um sistema de controle de processos. Este trabalho contribui na comprovação da possível utilização de medidas de capacitância para controle de processos.

\section{AGRADECIMENTOS}

Os autores agradecem à Fundação Araucária pelo auxílio financeiro.

\section{NOMENCLATURA}

$\begin{array}{ll}\varepsilon_{\mathrm{G}} & \begin{array}{l}\text { Saturação de gás } \\ \mathrm{U}_{\mathrm{G}}\end{array} \\ \mathrm{n} & \begin{array}{l}\text { Velocidade superficial de gás } \\ \left(\mathrm{m} \cdot \mathrm{s}^{-1}\right) \\ \text { fator dependente do regime do } \\ \text { fluxo }\end{array} \\ \alpha & \text { Nível de significância }(\%) \\ \mathrm{K} & \text { Constante dielétrica }\end{array}$

\section{REFERÊNCIAS BIBLIOGRÁFICAS}

AJBAR, A.; AL-MASRY, W.; ALI E.; (2008), "Prediction of flow regimes transitions in bubble columns using passive acoustic measurements". Chemical Engineering and Processing, 48(1), 101-110.

AKITA, K; YOSHIDA, F. (1973) "Gas holdup and volumetric mass transfer coefficient in bubble columns", Industrial and Engineering Chemistry Process Design and Development, 12(1), 76-82.

CUNHA FILHO, J. S. (2010), Estudo Experimental de Escoamento Bifásico em Tubo Circular Inclinado Usando Técnicas Ultrassônicas e de Visualização, COPPE/UFRJ, Rio de Janeiro - RJ (tese de doutorado), 260p.

DIONÍSIO, R.P. (2008), Simulação Tridimensional de uma Coluna De Bolhas - Diferentes Abordagens Geométricas e Modelagem, Unicamp, Campinas - SP (dissertação de mestrado), 79p.
KANTARCI, N.; BORAKB, F.; ULGEN K.O. (2004), "Bubble Column Reactors". Process Biochemistry, 40(7), 2263-2283.

LIRA, C.A.B.O (1980), Determinação de parâmetros de bolhas em escoamentos bifásicos, UFPE, Recife - PE (dissertação de mestrado), 55p.

MOORE, D. (2000), A Estatística Básica e Sua Prática, Editora LTC, Rio de Janeiro - RJ, p. 448.

MOREIRA, M. F. P.; FREIRE, J. T. (2003) "Influence of Gas and Liquid Flow Rates and the Size and Shape of Particles on the Regime Flow Maps Obtained in Concurrent Gas-Liquid Downflow and Upflow through Packed Beds". Industrial and Engineering Chemistry Research, 42(4), 929-936.

MUSSOI, F. L. R.; VILLAÇA, M. V. M. (2000), Capacitores, CEFET-SC, 39p.

SCHUMPE, A.; DECKWER, W.-D (1982), "Gas holdups, specific interfacial areas, and mass transfer coefficients of aerated carboxymethyl cellulose solutions in a bubble column", Industrial and Engineering Chemistry Process Design and Development, 21(4), 706-711.

SHAWAQFEH, A. T. (2002), "Gas holdup and liquid axial dispersion under slug flow conditions in gás-liquid bubble column". Chemical Engineering Science, 42(10), 767-775.

SILVA, L. F. L. R. (2008), Desenvolvimento de Metodologias para Simulação de Escoamentos Polidispersos usando Código Livre, COPPE/UFRJ, Rio de Janeiro - RJ (tese de doutorado), 262p. 